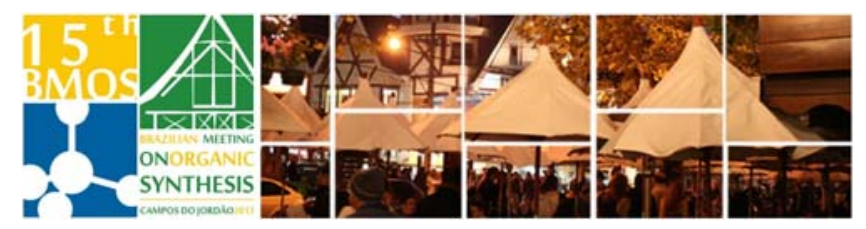

\title{
Enantioselective Heck Reactions with Aryldiazonium Salts. Challenges and Synthetic Opportunities
}

\section{Carlos Roque Duarte Correia}

\author{
Chemistry Institute - State University of Campinas, São Paulo - Brazil
}

*e-mail: roque@iqm.unicamp.br

Keywords: catalysis, Heck reactions, arenediazonium salts

\section{Abstract Speech}

Enantioselective catalysis has revolutionized the field of organic synthesis and its continuous development has brought huge benefits for society in general. In particular, the enantioselective arylation of olefins (Heck reaction) has been a subject of intense studies because of its potential for generating enantiomeric enriched drugs, fragrances and new materials which are in general more selective and therapeutically or environmentally less toxic. In this context, the palladium catalyzed coupling of arenediazonium salts to olefins (HeckMatsuda reaction) represents a practical and robust method to access structurally complex and bioactive molecules. The Heck-Matsuda arylations can be performed under aerobic conditions without requiring toxic phosphine ligands. Moreover, the HeckMatsuda arylations are much easier to carry out than conventional Heck protocols. The first example of arenediazonium salts as arylating agents of olefins catalyzed by palladium was described by Tsutomu Matsuda in 1977. However, in spite of the many advantages and the long-term existence of this reaction, its enantioselective version has constituted a considerable challenge for many decades due basically to the incompatibility between the available phosphine ligands and the arenediazonium salts.

In this lecture, some protocols developed to perform effective and practical asymmetric Heck-Matsuda reactions employing chiral, non-racemic, bisoxazoline ligands will be presented. ${ }^{1,2}$ Recent developments of this new enantioselective arylation reaction will also be highlighted.

Desymmetrizations:

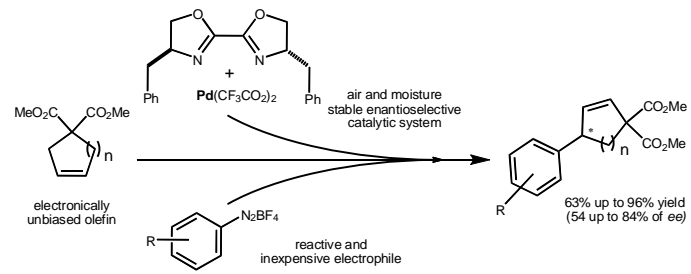

With acyclic olefins:

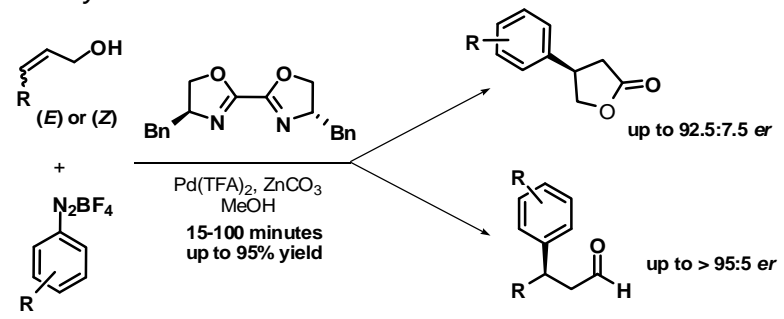

Acknowledgements: We thank the Brazilian funding agencies FAPESP, CNPq and CAPES for financial support.

\section{REFERENCES}

${ }^{1}$ Correia, C. R. D.; Oliveira, C. C.; Salles Jr., A. G.; and Santos, E. A. F. "The first examples of the enantioselective Heck-Matsuda reaction: arylation of unactivated cyclic olefins using chiral bisoxazolines" Tetrahedron Lett. 2012, 53, 3325-3328.

${ }^{2}$ Oliveira, C. C. Angnes, R. A and Correia, C. R. D. "Intermolecular Enantioselective Heck-Matsuda Arylations of Acyclic Olefins. Application to the Synthesis of $\beta$-Aryl- $\gamma$-Lactones and $\beta$-Aryl Aldehydes" J. Org. Chem. 2013, 78, 4373-4385. 